\section{MFA werden zu Software-Fachkräften}

Der Software-Anbieter medatixx bietet Praxisteams einen besonderen Service: Medizinische Fachangestellte (MFA) können sich bei der Niederlassung Mitte des Unternehmens in Walluf zur zertifizierten medatixx-Fachkraft ausbilden lassen. Ziel sei es, dass die MFA bei PCProblemen zunächst selbst eingreifen können. Das soll die Abläufe in den Praxen verbessern und den Ärzten Service-Kosten sparen. Vermittelt würden nicht nur Tricks und Kniffe rund um die medatixx- und MS-Office-Programme, sondern auch Grundlagen zu Hardware und Technik (www.medatixx.de).

\section{One-Click-Abrechnung erfolgreich gestartet}

Der E-Health-Anbieter Deutsches Gesundheitsnetz (DGN) hat gemeinsam mit der KV-Telematik-ARGE, den KVen und dem Software-Anbieter InterData die One-ClickAbrechnung getestet. Wie DGN mitteilt, haben im vierten Quartal 2011 fünf Arztpraxen mit der KV-Connect genannten Anwendung ihre Abrechnungsdaten erfolgreich an die KV übermittelt. Die Anwendung läuft zwar über den KV-SafeNet-Zugangsrouter DGN GUSbox S, die Praxen können aber die Daten mit einem Klick direkt aus der Praxis-Software an die KV senden und müssen sich nicht mehr extra auf einem Webportal einloggen. Ab dem zweiten Quartal 2012 soll die Anwendung allen GUSbox-Nutzern zur Verfügung stehen.

\section{Neue Software für vernetzte Ärzte}

Das Berliner Unternehmen Epikur Software \& IT-Service hat eine neue Version seiner Software epikur medico für die Verwaltung von Arztpraxen. Diese erlaubt mit moderner Netzwerktechnik den Zugriff auf Patientendaten von verschiedenen Standorten aus. Das Programm eigne sich daher besonders für überörtliche Praxisgemeinschaften und MVZ, heißt es in einer Pressemitteilung. Auch mobil könne auf die Praxisdaten sowohl mit dem Notebook (gesicherte VPN-Verbindung) als auch webbasiert mit dem iPad (SSL-Verschlüsselung) zugegriffen werden. Kalendereinträge ließen sich zudem mit dem Smartphone synchronisieren.

Epikur entwickelt seit 2006 Praxisverwaltungssysteme, die betriebssystemunabhängig unter Apple, Windows und Linux laufen. der Hewlett-Packard (HP) 14-Zoll Privacy Screen rund $56 €$. Aber auch die Varianten für 19-Zoll-Monitore, die in den Praxen gängiger sein dürften, sprengen nicht gleich das Budget: Der Kensington LCD-Sichtschutzfilter 19-Zoll liegt etwa bei $84,99 €$, der 15-Zoll LaptopSichtschutzfilter bei 60,99€. Etwas günstiger ist der 19-Zoll Targus Privacy Filter, der 79,90 € kostet. Für den 3M Privacy Filter 15,6-Zoll müssen Praxen rund $85 €$ zahlen. Es gibt aber auch von hsw3000 (4ProTec Blickschutz), Griffin, Dicota und Crumpler Sichtschutzfilter. Dabei gewähren alle Filter einen Einsichtswinkel von 30 Grad, die Wahl des richtigen Filters hängt also eher von Preis und Zusatznutzen ab. Einige Filter reduzieren nämlich gleichzeitig auch die Blendwirkung und die statische Aufladung des Bildschirms und damit die Staubablagerung. Schaden kann es zudem nicht, wenn auf ISO-Standards und das Erfüllen von Arbeitsschutzrichtlinien geachtet wird.

Wer sich eine Übersicht verschaffen will oder nach günstigeren Preisen sucht, sollte auch einen Blick auf die Websites von Hardware-Händlern wie Bechtle (www.bechtle.de) werfen.

Rebekka Höhl

\title{
Ärzte bestätigen Nutzen von Healthcare-IT
}

\section{Eine Studie zeigt: Für die Mehrzahl der Ärzte verbessert der IT-Einsatz die Patientenversorgung.}

$\mathrm{D}$ er Einsatz von Healthcare-IT - zum Beispiel in Form von elektronischen Patientenakten - bringt nach Ansicht der Mehrzahl der Ärzte Vorteile. So sind 68\% der Ärzte davon überzeugt, dass die IT organisationsübergreifende Prozesse verbessert. Zwei Drittel glauben, dass sie hilft, medizinische Fehler zu reduzieren. Herausgefunden hat das die Beratungsfirma Accenture in München. Das Unternehmen hat 3.700 Ärzte aus acht Ländern zum Nutzen von Healthcare-IT für ihren Praxisalltag befragt. Dabei zeigte sich im Vergleich der acht Länder - neben Deutschland waren es die USA, Frankreich, Spanien, Großbritannien, Australien, Kanada und Singapur - dass deutsche Ärzte in Sachen Healthcare-IT recht

\section{Was Healthcare-IT leisten kann}

Gefragt, welchen Nutzen der iT-Einsatz in der Praxis und Klinik bringt, antworteten $₫$ rzte:

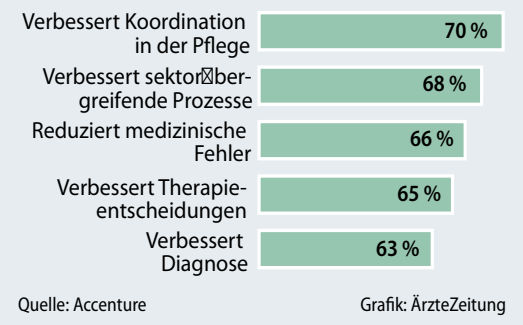

optimistisch sind. In der Durchschnittswertung zu allen Fragen über den Nutzen der IT im Praxisalltag erreichten deutsche Mediziner Rang drei. Genauer sehen hierzulande rund $62 \%$ der befragten Ärzte einen Nutzen für Praxis und Patienten, in Singapur sind es $64 \%$ der Ärzte und Spitzenreiter ist Spanien, wo über $70 \%$ der Ärzte einen klaren Nutzen erkennen können.

Dabei liegt nach Angaben deutscher Ärzte der größte Nutzen der HealthcareIT darin, dass sie hilft, organisationsübergreifende Prozesse zu verbessern. Zwei Drittel der deutschen Ärzte glauben aber auch, dass mithilfe der IT Diagnoseentscheidungen verbessert werden. Und $57 \%$ meinen, der IT-Einsatz reduziere die Zahl unnötiger Eingriffe. Eine Einschätzung, mit der deutsche Ärzte nicht allein sind, denn das sehen auch 55\% der Befragten aller Länder so.

Andere Vorteile, die die befragten Ärzte aus den acht Ländern bestätigten: Über $60 \%$ sagen, der IT-Einsatz verbessere den Genesungserfolg für Patienten, und rund $58 \%$ berichten, die IT würde den Patienten einen schnelleren Zugang zu Gesundheitsleistungen ermöglichen. Fast $70 \%$ der Ärzte geben an, dass elektronische Patientenakten und Co. die sektorenübergreifende Koordination von Pflege- und anderen Leistungen verbessere.

RebekkaHöhl 